\title{
AN INTERACTION-AWARE PREDICTIVE MOTION PLANNER FOR UNMANNED GROUND VEHICLES IN DYNAMIC STREET SCENARIOS
}

\author{
Junxiang Li, ${ }^{*}$ Bin Dai, ${ }^{* *}$ Xiaohui Li, ${ }^{*}$ Ruili Wang, ${ }^{* * *}$ Xin Xu, ${ }^{*}$ Bohan Jiang, ${ }^{*}$ and Yi Di****
}

\begin{abstract}
An interaction-aware predictive motion planning method for unmanned ground vehicles is presented in dynamic street scenarios. Although trajectory prediction in motion planners is widely covered in the past few years, most of them only consider the physical model of the vehicles and ignore the interaction among vehicles. Our motion planner predicts the future trajectories of surrounding participant vehicles taking the traffic interaction and manoeuvres into consideration. Furthermore, the motion planner exploits an improved trajectory generation method. The kinematically feasible trajectories are generated, which prevents a long-term collision using the predicted results in a probabilistic manner. The results show that our motion planner improves the safety and smoothness of driving trajectories in interactive scenarios.
\end{abstract}

\section{Key Words}

Predictive motion planner, interaction-aware motion prediction, manoeuvre-based trajectory prediction, unmanned ground vehicles, trajectory-generation approach

\section{Introduction}

Unmanned ground vehicles (UGVs) have been seen as the future of transportation. They are believed to have great potential to reduce traffic accidents, improve the efficiency

* College of Intelligence Science and Technology, National University of Defense Technology, Changsha 410073, People's Republic of China; e-mail: junxiangli90@gmail.com, xiaohui lee@outlook.com, xuxin_mail@263.net, birmingham001@ foxmail.com

** Unmanned Systems Research Center, National Innovation Institute of Defense Technology, Beijing 100091, People's Republic of China; e-mail: bindai.cs@gmail.com

*** Institute of Natural and Mathematical Sciences, Massey University, Auckland, New Zealand; e-mail: r.wang@massey.ac.nz

**** Information and Engineering School, Wuchang University of Technology, Wuhan, Hubei, People's Republic of China; e-mail: diyi8710@163.com

Corresponding author: Xin Xu

Recommended by Dr. Daqi Zhu

(DOI: 10.2316/J.2019.206-0072) of transportation systems, and provide a preferable option for people who are unable to drive [1], [2].

When UGVs are used in realistic road traffic, they need to handle various complex tasks, such as vehicle following, lane changing, merging, and interacting with other vehicles while complying with traffic rules. Although end-to-end learning shows great potential to be applied to UGVs [3], the conventional framework is widely used because of its reliability. The framework refers to a high-performance systematic integration of the following components: perception, localization, decision-making, planning, and control. The perception and localization module provides the real-time sensing information of surroundings and the current pose of a UGV. The behaviour reasoning module employs a reference path and online sensing information to generate discrete decisions (e.g., a lane change, a stopping behind a stopping line) and estimate the maximal allowed velocity of a UGV. The hierarchical planning framework has been widely used for the planning systems of UGVs [4], namely, global planning and real-time local planning. The global planner computes an optimal global route using a digital map either online or offline based on a given mission. The local planning is also known as the motion planning. Using the decisions generated by the behaviour reasoning module, the motion planner generates a kinematically feasible trajectory, which contains a spatial path and a velocity profile. Then, the trajectory tracking controller generates the steering and throttle/brake control inputs to follow the planned trajectory.

\subsection{Motivation}

Thus far, various techniques are presented to develop motion planners [5]-[8]. Although many traditional motion planners can ensure a UGV to drive autonomously in simple environments without traffic interaction, the ability to drive interactively in complex traffic scenarios still needs to be improved. Normally, the UGV on road may encounter various types of traffic participants such as cars and pedestrians. To plan safe and smooth trajectories, it is important to predict the future 
motion of surrounding participants. For simplicity, this paper describes motion planning without motion prediction for surrounding participants as non-predictive motion planning. Correspondingly, predictive motion planning refers to the one with motion prediction for surrounding participants.

Briefly, the current challenges of developing a motion planner for a UGV involve (1) understanding and accurately forecasting the motion of other participant vehicles (PVs); (2) interacting with dynamic environments based on the predictions. The related methods of motion prediction are discussed in Section 2. Traditional methods model the motion based on either kinematic/dynamic of vehicles or possible driving intention with the consideration of the historical trajectories. The interaction between traffic participants, especially PVs, is ignored. Interaction-aware motion prediction has an insight into the whole traffic situation. However, the interaction is difficult to be modelled effectively [9]. Moreover, to the best of our knowledge, the motion prediction of PVs is mostly used to improve the capacity of intelligent decision-making of a UGV or assess criticality. There seems to be little research on predictive motion planning. However, motion planning directly influences the smoothness of driving trajectories. Therefore, the research on interaction-aware predictive motion planning is worthwhile.

\subsection{Contributions}

To address the challenges, this paper proposes an interaction-aware predictive motion planner (IAPMP) for UGV in dynamic street scenarios. It can interactively predict the future trajectories of PVs based on their predicted manoeuvres. Besides that, it also generates kinematically feasible trajectories and avoids the long-term collision in a probabilistic manner. The main contributions of this paper include two aspects.

(1) Our novel prediction method integrates the driving intention, vehicle physics and traffic interaction (mainly collision avoidance) into account in the trajectory prediction, making long-term prediction more accurately in complex scenarios.

(2) Our planning method generates tractable and interaction-aware trajectories. The experimental results showed that the planned trajectories of our motion planner are smoother and more human-like than those of integrated local trajectory planning (ILTP) [10] in some typical street scenarios.

\subsection{Layout}

The paper is organized as follows. Section 2 summarizes the state-of-the-art motion planning techniques for UGVs. In Section 3, specific details of our predictive motion planning, based on the interaction-aware trajectory prediction, are introduced. Section 4 reports the experimental set-up and results. In Section 5, conclusion and future work are discussed.

\section{State of the Art}

\subsection{Non-predictive Motion Planning}

As discussed in Section 1, a motion planning algorithm plays a significant role in robotics. The history of the research on motion planning can be traced over the last few decades to DARPA (Defense Advanced Research Projects Agency) Urban Challenge. Since then, many approaches have been presented, which are commonly classified into two types [11]: graph traversal-based approaches and trajectory generation-based approaches.

The approaches based on graph traversal are only appropriate for UGVs travelling at relatively low speed, such as the state lattice search in [12] and [13], Hybrid $\mathrm{A}^{*}$ in [14], the path segments search in [15], the randomsampling-based approach (such as RRT and RRT variants) in [16]-[18]. Besides, most of these methods generate paths connected by pre-computed path primitives without considering motion continuity. Consequently, they are more likely to cause unsmooth movements.

The approaches based on trajectory generation are proposed with consideration of modelling vehicle kinodynamics. According to [4], these approaches can be divided into control-space approaches and state-space approaches according to different sampling methods in the approaches. The former can satisfy the constraints of vehicle kinematics, but not the environmental constraints like road geometries. Conversely, the latter can guarantee the satisfaction of environmental restrictions, whereas more computing resource is required during the generation process.

The control-space generation approach refers to parameterizing the control space through arcs and clothoids, then each sampling parameterized control (i.e., control input) can generate a trajectory. This approach (e.g., in [19]-[21]) can guarantee that the generated trajectories are kinematically feasible and easily tracked by a controller. Therefore, the control-space trajectorygeneration approach is an effective approach when there are no road constraints. But, this approach is ineffective when environmental constraints (e.g., road geometries) are considered.

The state-space generation approaches obtain the expected states according to road geometries and then generate the trajectory connecting the current state and the expected state. An inverse generation method using polynomial spirals to ensure smooth execution is developed in [22]-[24] and applied to urban roads in [25]. However, the complex iteration process is likely to affect the effectiveness of the planner and causes high computing complexity. The motion planner proposed in [26] generates a reference path based on the road centreline in the Frenét frame, making the lateral and longitudinal movements consistent with the road shape. Similarly, Gu et al. [27]-[29] propose a framework that executes a curvature reduction on the reference path to generate a smooth trajectory. In [30], the polynomial trajectory segment is iteratively extended to pass through designated waypoints. Recently, a new generation approach based on a quartic Bézier curve is proposed [31], [32]. Our previous work in [10], [33], [34] 
designs a framework (ILTPTC) for UGVs, which also contains a state-space generation-based motion planner.

\subsection{Predictive Motion Planning}

Although non-predictive motion planners can enable a UGV to drive autonomously with consideration of safety in static environments, the ability to drive interactively in complex traffic scenarios still needs to be improved. An improved method is predicting the future motion of surrounding PVs.

The state-of-the-art techniques of motion prediction can be classified into three levels according to the complexity of the motion model [9]. (1) The elementary level is physics-based approaches, which only model the motion depending on kinematics and dynamics of vehicles (e.g., the constant velocity $(\mathrm{CV})$ and the constant acceleration $(\mathrm{CA})$ [35]-[38]). (2) The intermediate level is manoeuvre-based approaches. In this layer, how the future motion depending on manoeuvres performed by drivers is also considered in the motion model. Common approaches use classification algorithms (e.g., Naive Bayes algorithm [39], support vector machine [40], dynamic Bayesian network [37], [41], [42], hidden Markov model [43], and deep neural network $[44],[45])$ to classify driver manoeuvres. The manoeuvre classification is also called as intention recognition. (3) The most complex level is interaction-aware approaches, which also deal with how the motion is influenced by the traffic interaction (e.g., collision avoidance, social conventions and traffic rules). Interaction-aware approaches have an insight into the whole traffic situation. However, there are few research based on interaction-aware approaches according to the review [9]. Besides, most of the related approaches (e.g., Dynamic Bayesian Network [46]-[48]) only consider the interaction in the intention recognition, not in the trajectory prediction.

As far as we know, the motion prediction for PVs is mostly applied in the decision-making of UGVs. One of prediction-based decision-making approaches is based on the partially observable Markov decision process (POMDP) [49]-[52]. An attractive advantage of the POMDP-based approaches is that decision results are theoretically optimal. However, the computational complexity of abstracting the interaction into a POMDP model increases exponentially with the number of traffic participants and decisions. Lee and Kim [53] try to use a massively parallel algorithm to calculate POMDP. It has an outstanding performance compared to the existing CPU-based algorithms on some chosen benchmarks. Another example of prediction-based decision-making approaches is the game-theory-based approach. An integrated framework for interactive prediction and decision making based on Game Theory is exploited in [54]. This framework successfully predicts the merging intention of a most relevant car in a highway. However, the two-player game-theory-based algorithm only deals with pairwise dependence and hardly extends in complex scenarios. In [55], a cooperative algorithm, which exploits Monte Carlo Tree Search as a decision model, is proposed. The evaluation with the highest scores along the tree is used to find the lateral and longitudinal actions for UGVs. Overall, because of high expense and difficulty in building an accurate probabilistic state transition distribution, the extensibility of prediction-based decision making is limited. Moreover, the calculated discretized decision may conflict with the planning trajectories, as the safety and feasibility of the manoeuvre cannot be calculated in the decision making.

Oppositely, motion planning directly influences the safety and feasibility of driving trajectories. Utilizing predicted results in motion planning is crucial for modelling interactions. However, there seems to be little research on interaction-capable motion planning except for the following. Damerow and Eggert [56] present a so-called predictive risk map, which measures the risks of different manoeuvres. Then, the RRT* algorithm is used to find an optimal trajectory with the lowest risk. In [57], [58], a prediction- and cost function-based algorithm and its intention-integrated version are proposed to enable a UGV to implement socially compliant motion planning. Similar to our method, the methods combine a cost-function-based driving strategy with a prediction model. However, the assumption for the prediction model seems too idealistic. The prediction model assumes that the future motion of each vehicle is keeping a pre-computed minimum safety distance to a leading vehicle. Moreover, the weight parameters of the cost function far exceed the number of ours, thus leading to complex parameter adjustment.

\section{Method}

The framework of our IAPMP is shown in Fig. 1. IAPMP has four components in total: (1) an interaction-aware trajectory predictor, (2) a path planner, (3) a performance evaluator and (4) a velocity planner.

The entire process of our motion planner runs periodically in a short planning cycle. At first, the interactionaware trajectory predictor predicts possible future states of other dynamic PVs. Then, the path planner samples terminal states (i.e., positions, headings, curvatures) of each planning cycle adaptively, based on road geometries. Multiple kinematically feasible paths (or candidate paths) are generated from an initial state to the terminal states. After that, the performance evaluator, which evaluates the long-term safety and smoothness for each candidate path, is built to select the optimal path. Finally, based on the length, the curvature of the optimal path and the maximal allowed velocity from the behaviour reasoning module, the velocity planner generates executable velocity profiles.

\subsection{Interaction-Aware Trajectory Prediction}

The object of the trajectory prediction in this section is PVs, not a UGV. As experienced human drivers, the prediction for PVs provides us enough time to prepare and make suitable decisions in advance. Accurate long-term trajectory prediction relies on the intention recognition, but the research on intention recognition itself (discussed in Section 2.2) is nontrivial and independent of the trajectory prediction. Our previous research on intention recognition of PVs can be found in [59]. Therefore, this paper mainly 


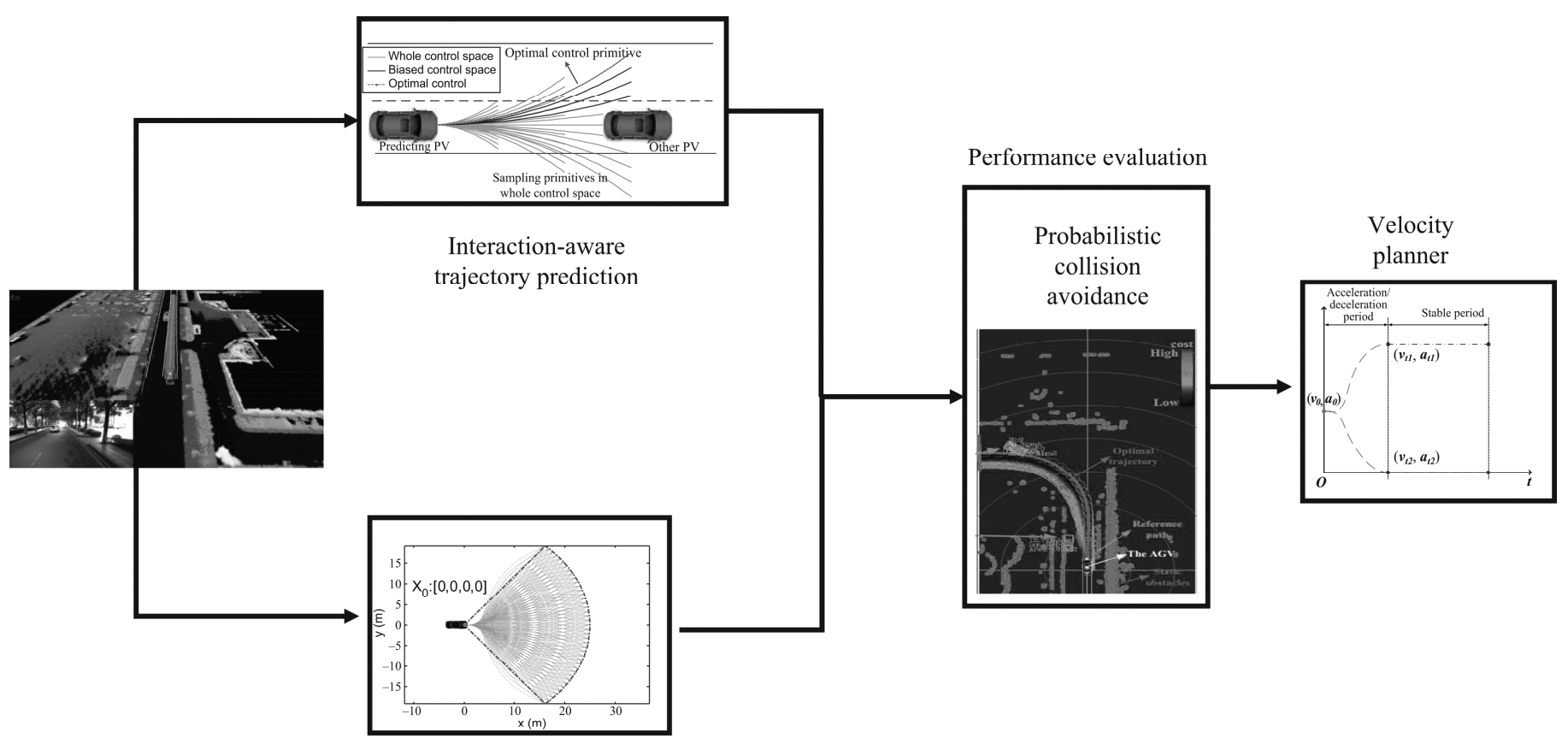

Path planner

Figure 1. Framework of IAPMP.

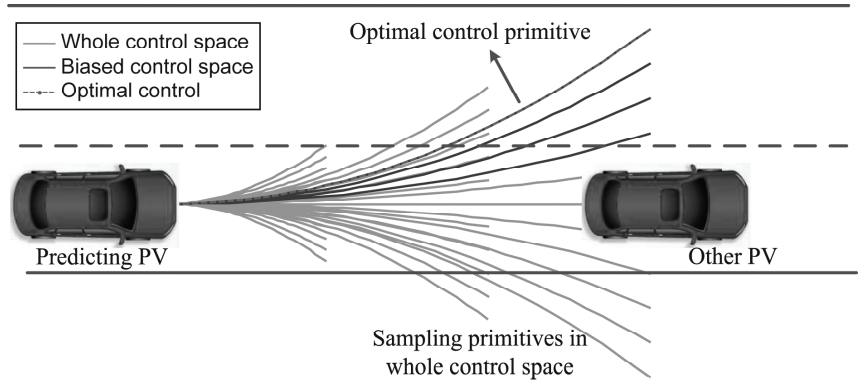

Figure 2. The process of proposed manoeuvre prediction method.

focuses on the interaction-aware trajectory prediction and assumes the intention of PVs can be recognized accurately in the simulation experiment. The recognized intention contains the lane change (e.g., changing to the left lane) and the velocity change (e.g., accelerating).

The interaction-aware trajectory prediction includes two steps: manoeuvre prediction and trajectory prediction. (1) Manoeuvre prediction predicts specific longitudinal and lateral manoeuvre values (i.e., linear and angular acceleration) which constitute the optimal manoeuvre with consideration of the traffic interaction (i.e., collision avoidance). We assume that a normal and reasonable driving manoeuvre will follow the intention and avoid collisions. The process of the manoeuvre prediction for one PV is shown in Fig. 2. (2) Trajectory prediction is defined as the prediction of the possible state distribution along the optimal manoeuvre. The state of a PV includes the position coordinate, the heading, the angular velocity and the linear velocity. Concretely, the process is introduced below.

First, based on the recognized intention (i.e., lane changing and velocity changing), a biased manoeuvre space will be chosen in the whole control space $U_{s}$. The whole control space, which is defined as (1), is a Cartesian product of two sets. These two sets are composed of a set of lateral manoeuvre space and a set of longitudinal manoeuvre space. The elements of the set are scopes of lateral or longitudinal acceleration, which are also called the biased manoeuvre space. In terms of lane changing, three intentions (left-lane changing, right-lane changing and lane keeping) correspond to different scopes of the angular acceleration $\left(\tilde{w}_{L C}, \tilde{w}_{R C}\right.$ and $\tilde{w}_{K L}$, respectively). Similarly, velocity-changing intentions correspond to different scopes of the linear acceleration/deceleration $\left(\tilde{a}_{A c c}\right.$, $\tilde{a}_{D c c}$ and $\tilde{a}_{C o n s t}$, respectively):

$$
\begin{gathered}
U_{s}=\left\{\tilde{w}_{L C}, \tilde{w}_{R C}, \tilde{w}_{K L}\right\} \times\left\{\tilde{a}_{A c c}, \tilde{a}_{D c c}, \tilde{a}_{C o n s t}\right\} \\
\tilde{w} \in\left[\tilde{w}_{\min }, \tilde{w}_{\max }\right] \subset\left\{\tilde{w}_{L C}, \tilde{w}_{R C}, \tilde{w}_{K L}\right\} \\
\tilde{a} \in\left[\tilde{a}_{\min }, \tilde{a}_{\max }\right] \subset\left\{\tilde{a}_{A c c}, \tilde{a}_{D c c}, \tilde{a}_{\text {Const }}\right\}
\end{gathered}
$$

Then, the manoeuvre primitives $(\hat{u}=[\tilde{w}, \tilde{a}])$ will be sampled in the chosen biased manoeuvre space. The chosen biased manoeuvre space is the corresponding subsets of two sets, as (2). Each sampled driving manoeuvre primitive for a PV will generate a trajectory according to the kinematic model (3) of PVs. At time step $t$, a PV applied a manoeuvre primitive $\hat{u}_{t+1}$ will transit from the state $\hat{X}_{t}$ to the state $\hat{X}_{t+1}$.

$$
\begin{aligned}
p^{x} & =v * \cos (\phi) \\
p^{y} & =v * \sin (\phi) \\
\dot{\phi} & =\frac{v * \tan (\delta)}{L_{w}} \\
\dot{\delta} & =\tilde{w} \\
\dot{v} & =\tilde{a}
\end{aligned}
$$


where $\hat{X}_{t}=\left[p_{t}^{x}, p_{t}^{y}, \phi_{t}, \delta_{t}, v_{t}\right]^{\prime}$ and $\hat{u}_{t+1}=\left[\tilde{w}_{t+1}, \tilde{a}_{t+1}\right]^{\prime}$; $\left(p^{x}, p^{y}\right)$ means the position coordinate $\mathbb{P} ; \phi, \delta$ and $v$ mean the heading, angular velocity and linear velocity, respectively; $L_{w}$ is the estimated wheelbase parameter.

To consider the interaction with other vehicles, the optimal manoeuvre primitive, which generates the optimal trajectory, is chosen by a performance function. The performance function (4) penalizes the possible collision with other PVs (except itself) along trajectories. Several points of each trajectory, corresponding to a control primitive, are extracted for the cost calculation. After evaluating the trajectory, the highest-score manoeuvre primitive is chosen as the possible manoeuvre of the PV:

$\hat{u}_{t+1}^{*}=\arg \operatorname{man}_{u_{i}=1}^{u_{n}}\left(\min _{j=1 \ldots \psi, k=1 \ldots \varsigma}\left(\left\|\hat{X}^{i j}(1,2)-\hat{X}^{k}(1,2)\right\|\right)\right)$

where $i$ is the ordinal number of the manoeuvre primitive $u$, $u_{n}$ is the number of the manoeuvre primitive, $\psi$ represents the total extracted number in a trajectory, $\varsigma$ represents the number of other PVs and $\hat{X}(1,2)$ represents the position of PVs.

The trajectories are determined by current states $\left(\hat{X}_{t}\right)$ and future manoeuvres $\left(\hat{u}_{t+1}^{*}\right)$ of PVs. To predict the distribution of the states along the trajectory accurately, a continuous Bayes filter is used. The state equation is written as (5), where $\tilde{f}()$ is introduced in (3). The measurement equation is written as (6). The process noise $(m)$ and the measurement noise $(r)$ are assumed to be Gaussian distribution:

$$
\begin{aligned}
\frac{d \hat{X}_{t+1}}{d t} & =\widetilde{f}\left(\hat{X}_{t}, \hat{u}_{t+1}^{*}, t\right)+m, \quad m \sim \mathcal{N}(0, M) \\
Y_{k} & =C \hat{X}_{k}+r, \quad r \sim \mathcal{N}(0, R) \\
C & =\left[\begin{array}{lllll}
1 & 0 & 0 & 0 & 0 \\
0 & 1 & 0 & 0 & 0 \\
0 & 0 & 0 & 0 & 1
\end{array}\right]
\end{aligned}
$$

Because the kinematic system is a typical continuoustime nonlinear model, and the measurement system is a discrete model, a continuous-discrete unscented Kalman filter (CDUKF) [60] is used to predict future states.

\subsection{Path Planning}

Path planning aims to generate smooth and feasible paths for the low-level controller of a UGV complying with the environmental constraints. It contains adaptive sampling and model-predictive generation. To make the iteration process of generation faster, a pre-computed lookup table is designed to store the initial parameters.

First, the adaptive sampling algorithm [4] is exploited to sample the expected terminal states. The road geometry information, such as global route state $\left(X_{r e f}\right)$ and lane width $\left(w_{l}\right)$, is extracted from the perception system.
In addition, the vehicle width $\left(w_{v}\right)$ is also taken into consideration. During each sampling process, the terminal states are sampled at a distance from the position of the UGV along the global route (i.e., reference line). Note that the distance, which is called preview distance, can be tuned according to the current velocity. The terminal position coordinates are sampled along the reference line with multiple lateral offsets $\left(d_{o f f}\right)$. The headings of the sampling points are set to be the same as those of the reference line. The curvatures of the sampling points are set as follows:

$$
\kappa=\left(\kappa_{\text {ref }}^{-1}-d_{\text {off }}\right)^{-1}
$$

where $\kappa_{r e f}$ is the curvature of the reference line.

Then, the model-predictive generation method [61] is exploited for generating kinematically feasible paths. The curvature of the path $(\kappa)$ is modelled as a polynomial function as its continuity can avoid jerky turnings. To solve the parameters in $\kappa$, the curvature is modelled as a cubic polynomial. The state equation is derived as (8)

$$
\begin{aligned}
x\left(s_{f}\right) & =\int_{0}^{s_{f}} \cos \left[a s+\frac{b s^{2}}{2}+\frac{c s^{3}}{3}+\frac{d s^{4}}{4}\right] d s \\
y\left(s_{f}\right) & =\int_{0}^{s_{f}} \sin \left[a s+\frac{b s^{2}}{2}+\frac{c s^{3}}{3}+\frac{d s^{4}}{4}\right] d s \\
\phi\left(s_{f}\right) & =a s_{f}+\frac{b s_{f}^{2}}{2}+\frac{c s_{f}^{3}}{3}+\frac{d s_{f}^{4}}{4} \\
\kappa\left(s_{f}\right) & =a+b s_{f}+c s_{f}^{2}+d s_{f}^{3} \\
\left|\kappa\left(s_{f}\right)\right| & \leq \kappa_{\max }
\end{aligned}
$$

To solve these nonlinear equations, the parameters $\left\{b, c, d, s_{f}\right\}$ are iteratively calculated through Newton's method. Initial values for the parameters influence the convergence of iteration. Besides, a good initial value can prevent solution falling into a local minimum. Therefore, inspired by the work of [62]-[64], a pre-computed lookup table is presented to store initial values. As illustrated in Fig. 3, the initial values are determined by the parameters of pre-computed paths. The storing sequence

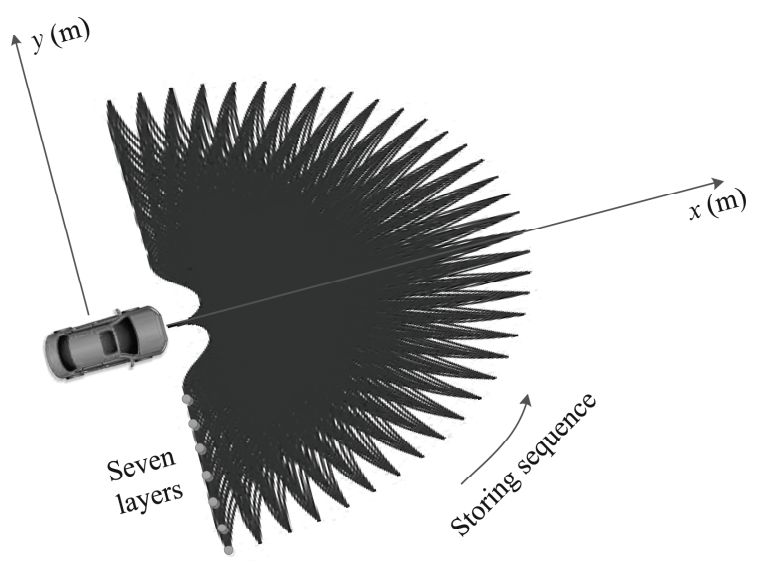

Figure 3. Visualized lookup table storing initial values. 
is anti-clockwise, based on the position of final sampling points from inside to outside. At each position, the storing sequence for different headings is also anti-clockwise.

\subsection{Performance Evaluation}

In this section, the final planned path is chosen by performance evaluation after a collision test. The evaluation exploits the predicted trajectories of PVs.

For the sake of safety, the collision test for static obstacles (e.g., road boundaries and traffic cones) is performed before the evaluation. The same number of points is extracted from each candidate path. Inspired by [10],[33],[65], each vehicle is approximated by a set of circles with the same radius. If the distance from the extracted point to the static obstacle is shorter than the threshold value, the path will be trimmed to the last eligible point.

Then, function (9) is designed to evaluate the performance of the remaining path. The optimal path will be chosen for minimizing the total cost. Note that each cost term is normalized to be within $[0,1]$

$i^{*}=\arg \min _{i=1}^{\Psi}\left(\mathbb{W} * \mathbb{C}^{T}\right)=\arg \min _{i=1}^{\Psi}\left(w_{o} C_{o}+w_{d} C_{d}+w_{s} C_{s}\right)$

where $\mathbb{C}=\left[C_{o}, C_{d}, C_{s}\right]$ is the cost vector; $\mathbb{W}=\left[w_{o}, w_{d}, w_{s}\right]$ is the tunable weight vector; $i$ and $\Psi$ are the index and the total number of candidate paths, respectively.

The cost $C_{o}$ evaluates the safety of the candidate path and reflects the proximity of the candidate path to the PVs. As the trajectories of the PVs are predicted in Section 3.1, a probabilistic penalty can be exploited. Note that the predicted position of the UGV is deterministic and can be obtained through the planned state at the corresponding time, which is $P_{U G V}=X_{t}(1,2), \Sigma_{t}^{i}=0$. But the predicted position of the PVs is uncertain and fits the Gaussian distribution, $P_{P V} \sim N\left(\hat{X}_{t+1}(1,2), P_{t+1}\right), \Sigma_{t}^{j}=P_{t+1}$. Then, the collision probability of the two vehicles ( $i$ represents UGV and $j$ represents PV), which complies with a bivariate Gaussian distribution, is

$$
P_{t}(i \otimes j)=\int_{\|p\|<2 r} \frac{\exp \left(-\frac{1}{2}\left(p-V_{t}^{i j}\right)^{\prime}\left(\Sigma_{t}^{i}+\Sigma_{t}^{j}\right)^{-1}\left(p-V_{t}^{i j}\right)\right)}{2 \pi\left|\Sigma_{t}^{i}+\Sigma_{t}^{j}\right|^{1 / 2}} d p
$$

where $V_{t}^{i j}=X_{t}(1,2)-\hat{X}_{t}(1,2)$, which is the relative position from the predicted position of the UGV to that of $j$ th $\mathrm{PV}$. The parameter $r$ is the approximate radius threshold of the collision. The result can be integrated numerically. For all PVs, the collision probability is modelled as the penalty:

$$
C_{o}=1-\prod_{j=1}^{m}\left(\prod_{t=1}^{\tau}\left(1-P_{t}(i \otimes j)\right)\right)^{1 / \tau}
$$

where $m$ is the number of PVs, $\tau$ is the number of time samples.
The cost $C_{d}$ penalizes the path deviation from the global route

$$
C_{d}= \begin{cases}\frac{1}{N} \sum_{i=1}^{N} \frac{D_{i}^{2}}{D_{\max }^{2}} & \text { if } D_{\max } \neq 0 \\ 0 & \text { if } D_{\max }=0\end{cases}
$$

where $N$ is the number of the extracted points from each path, $D$ is the deviation distance of the discrete point from the global route, $D_{\max }$ is the maximal deviation distance among all $D_{i}$ in each planning cycle.

The $\operatorname{cost} C_{s}$ reflects the path smoothness, which affects the riding comfort:

$$
C_{s}= \begin{cases}\frac{1}{2 * N} \sum_{i=1}^{N}\left(\frac{\kappa_{i}^{2}}{\kappa_{\max }^{2}}+\frac{{\dot{\kappa_{i}}}^{2}}{\dot{\kappa}_{\max }^{2}}\right) & \text { if } \kappa_{\max } \neq 0 \wedge \dot{\kappa}_{\max } \neq 0 \\ 0 & \text { else }\end{cases}
$$

where $\kappa$ is the curvature of the extracted points in the candidate path; $\dot{\kappa}$ is the derivative of the curvature; $\kappa_{\max }$ is the maximal curvature among all $\kappa_{i}$ in each planning cycle and similarly, $\dot{\kappa}_{\max }$ is the maximal derivative of the curvature.

\subsection{Velocity Planning}

The velocity planning algorithm is introduced as follows. First, a maximal velocity limit is calculated according to driving constraints. Second, the desired velocity, related to the maximal velocity limit, is calculated. Third, a tractable velocity profile is designed.

For the sake of safety, a UGV needs to have a maximal velocity limit $\left(V_{\max }\right)$ to avoid a sudden brake in a realistic environment. $V_{\max }$ is restricted to be minimum of the following factors [34]: (1) maximal allowed velocity $\left(V_{\text {mal }}\right),(2)$ maximal lateral velocity $\left(V_{\text {mlat }}\right)$ and $(3)$ maximal longitudinal velocity $\left(V_{\text {mlon }}\right)$.

As we know, the desired velocity meets the basic condition of $V_{\text {desire }} \in\left[0, V_{\max }\right)$. Then, a univariate function (14) of $V_{\text {desire }}$ is designed. The function complies with the condition that the desired velocity has a positive correlation to the path length when the length is longer than the safe braking distance $\left(D_{\text {safe }}\right)$. With the path length approaching infinity, the desired velocity approaches $V_{\max }$ :

$$
V_{\text {desire }}= \begin{cases}\frac{2 V_{\max }}{1+\exp \left(D_{\text {safe }}-s\right)}-V_{\max } & \text { if } s \geq D_{\text {safe }} \\ 0 & \text { if } s<D_{\text {safe }}\end{cases}
$$

As Fig. 4 shows, the velocity profile which consists of two segments is designed. To ensure the continuous acceleration/deceleration, the cubic polynomials function (15) is exploited as the first segment. Note that the acceleration/deceleration needs to follow the constraints of maximal acceleration/deceleration. Then, a stable period which maintains the desired velocity follows

$$
v(t)=k_{0}+k_{1} t+k_{2} t^{2}+k_{3} t^{3}
$$




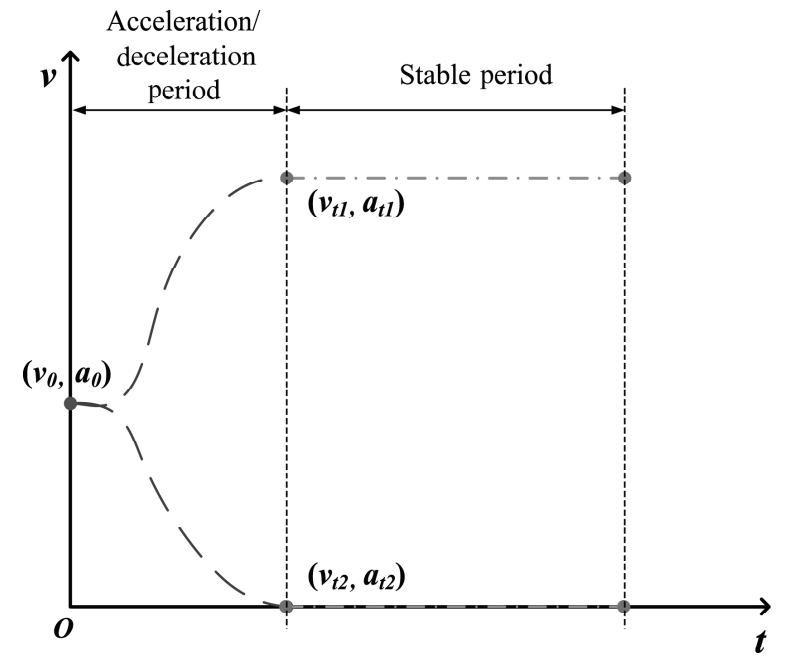

Figure 4. An example of velocity profile.

The current velocity $v_{0}$ can be acquired from the sensing information. The final velocity $v_{f}$ of the planned path, which is equal to the desired velocity, can be obtained by (14). Assuming that both initial acceleration $a_{0}$ and final acceleration $a_{f}$ are zero. The planned path length $s_{f}$ is in Section 3.2. Therefore, the unknown parameters $\left\{k_{0}, k_{1}, k_{2}, k_{3}\right\}$ in (15) and the planned timespan $t_{f}$ can be solved via the following equations:

$$
\begin{aligned}
v(0) & =k_{0}=v_{0} \\
v\left(t_{f}\right) & =k_{0}+k_{1} t_{f}+k_{2} t_{f}^{2}+k_{3} t_{f}^{3}=v_{f} \\
a(0) & =k_{1}=0 \\
a\left(t_{f}\right) & =k_{1}+2 k_{2} t_{f}+3 k_{3} t_{f}^{2}=0 \\
s\left(t_{f}\right) & =k_{0} t_{f}+\frac{1}{2} k_{1} t_{f}^{2}+\frac{1}{3} k_{2} t_{f}^{3}+\frac{1}{4} k_{3} t_{f}^{4}+s(0)=s_{f}-v_{f} * t_{s}
\end{aligned}
$$

where $t_{s}$ is the duration time of the velocity maintaining period.

\section{Experimental Results and Discussion}

In this section, two experiments are conducted. One experiment is to justify the accuracy of our interactionaware trajectory prediction. The other experiment is to verify the performance of predictive motion planning. The results of the second experiment are only presented through simulation as simulation is the best way to compare the approaches without the interference caused by the instability of other systems (e.g., control and perception system). The experiments are simulated in Prescan 8.0 and Matlab R2015b. Prescan is a high-fidelity simulation environment for the development of autonomous driving. The default path follower in Prescan is used to translate a planned trajectory into wheel steering angle input and velocity input for the controller. The planning cycle is $0.05 \mathrm{~s}$. The main parameters are listed in Table 1. Note that the parameter selection of the performance function can be tuned on a real UGV.

\subsection{Accuracy of Prediction}

As the predictor in a motion planner is critical for smooth and safe planning, two experiments on the accuracy of
Table 1

Main Parameters of Experiments

\begin{tabular}{|l|l|}
\hline Meaning & Value \\
\hline Parameter $r$ in $C_{o}$ & 2.5 \\
\hline Lane width $(\mathrm{m})$ & 3.5 \\
\hline Vehicle width $(\mathrm{m})$ & 1.86 \\
\hline Vehicle length $(\mathrm{m})$ & 4.45 \\
\hline Parameter $(\mathbb{W})$ in performance function & {$[0.8,0.1,0.1]$} \\
\hline
\end{tabular}

trajectory prediction are performed. One experiment is verified on real traffic data, while the other is verified in the simulation platform.

(1) Verification on Real Traffic Data: The qualitative accuracy of the proposed prediction method has been studied on real traffic data in a massive public dataset NGSIM I-80. It was collected from the traffic on Interstate highway 80 . The detailed descriptions of the dataset can be found in [59]. The trajectories in the dataset are collected by a visual detecting system. Thus, there is noise in the recorded trajectory.

For simplicity, we only consider the vehicles within the distance less than a fixed threshold from the predicting $\mathrm{PV}$ as interacting obstacles. The threshold is set to be $20 \mathrm{ft}$. Then, as the intention has been predicted in the previous research [59], our method of trajectory prediction can be directly exploited to each vehicle with the assumption of known intentions.

Predicting results are shown in Fig. 5. As shown in the figures, the predicted trajectory of our method can coincide better with the actual lane-change historical trajectory compared to the method using CV. This is particularly apparent in high-speed and smooth lane changing with an uncertain heading.

(2) Qualitative Evaluation on Simulation: We compare the following predictors: (1) a constant velocity predictor (CVP), (2) a trajectory-generation-based Kalman predictor (TKP) in KPMP [34] and (3) the proposed interaction-aware predictor (IAP) in IAPMP. The simulated street scenario is shown in Fig. 6. In this scenario, the UGV only predicts the trajectory of the target participant vehicle (TPV), which changes the lane to avoid the slower participant vehicle (FPV) in front. The presetting trajectories are shown in the figure. The recorded cycle is $0.05 \mathrm{~s}$ for predicted trajectories, and $0.1 \mathrm{~s}$ for presetting trajectories. Each predictor predicts the future trajectory of the TPV in $3 \mathrm{~s}$ ahead.

Figure 7 compares the predicted results of position at three time points (the 1st second, the 2 nd second and the 3rd second, respectively). During the lane change (Fig. 7(a) and (b)), IAP predicts a larger angular acceleration $\tilde{w}$ for TPV. As can be seen in Fig. 7(a), there is an obvious gap, which is marked in the box. Similarly, in Fig. 8(a) and (b), IAP predicts velocity changes. The results suggest that IAP intends to avoid the possible collision with the slower FPV in 


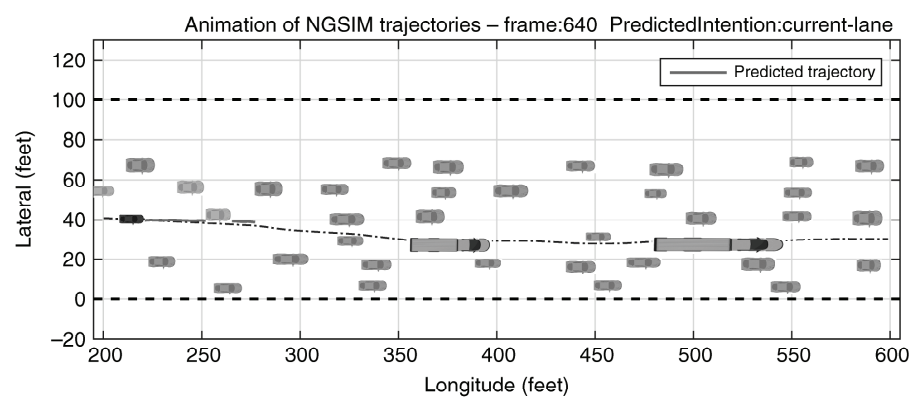

(a)

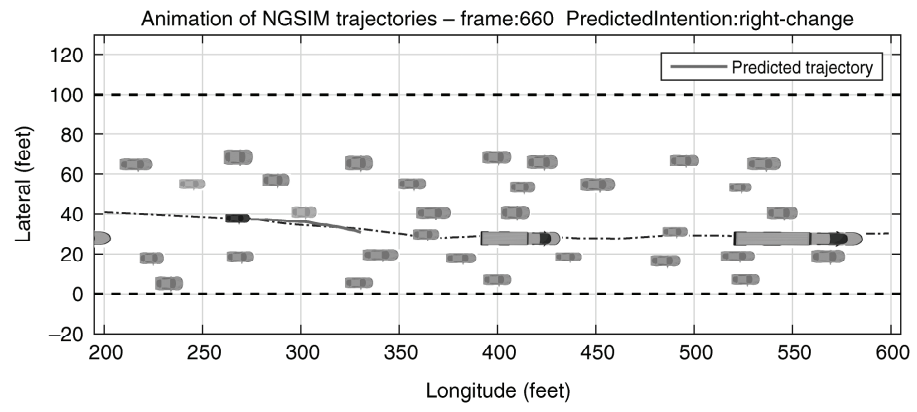

(c)

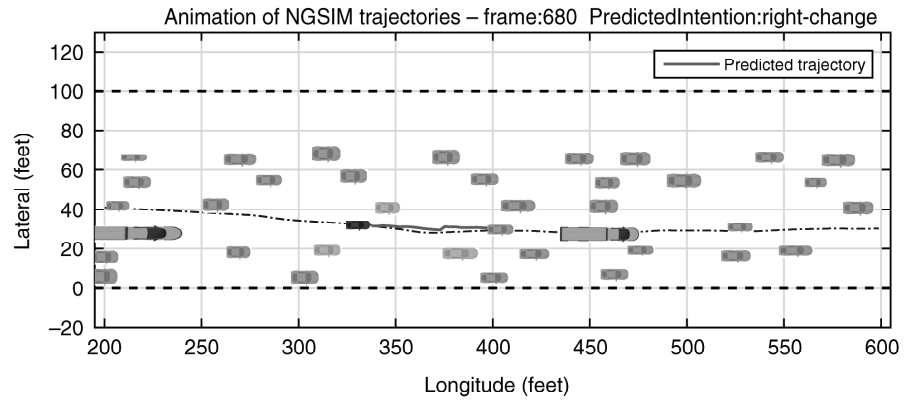

(e)

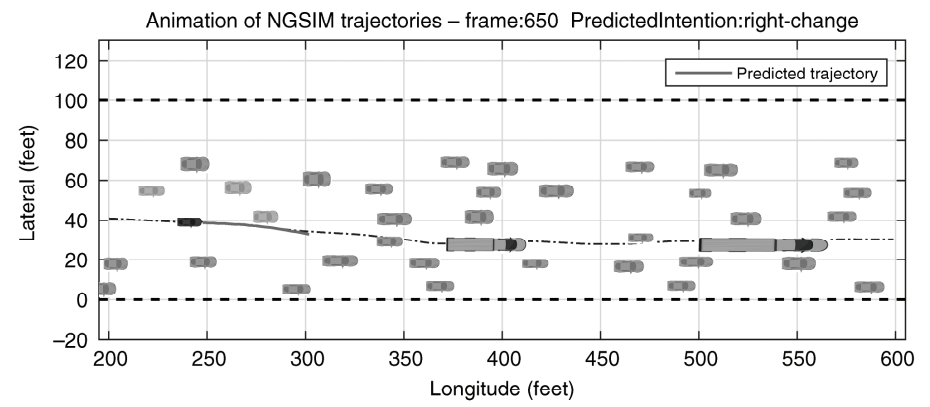

(b)

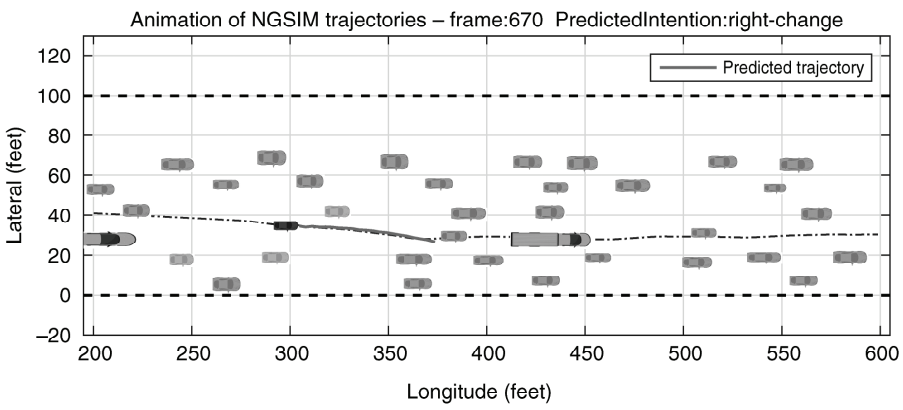

(d)

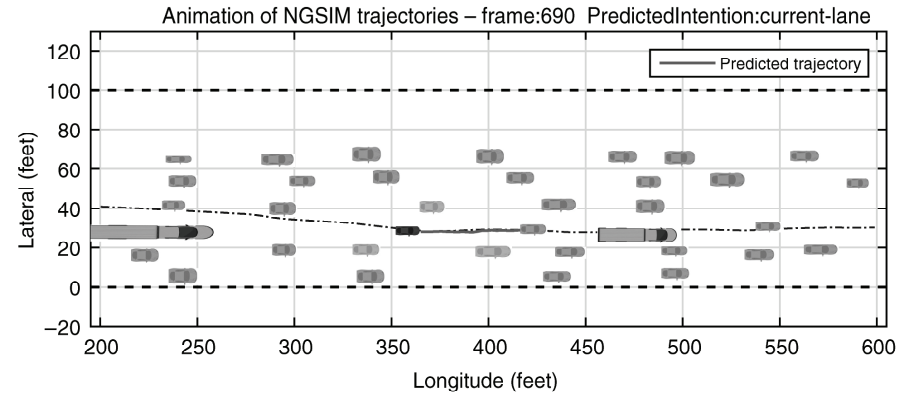

(f)

Figure 5. The predicted trajectory and actual trajectory of Vehicle \#121 in Dataset I-80: (a) frame $=640 ;(b)$ frame $=650$; (c) frame $=660$; (d) frame $=670$; (e) frame $=680$; and (f) frame $=690$.

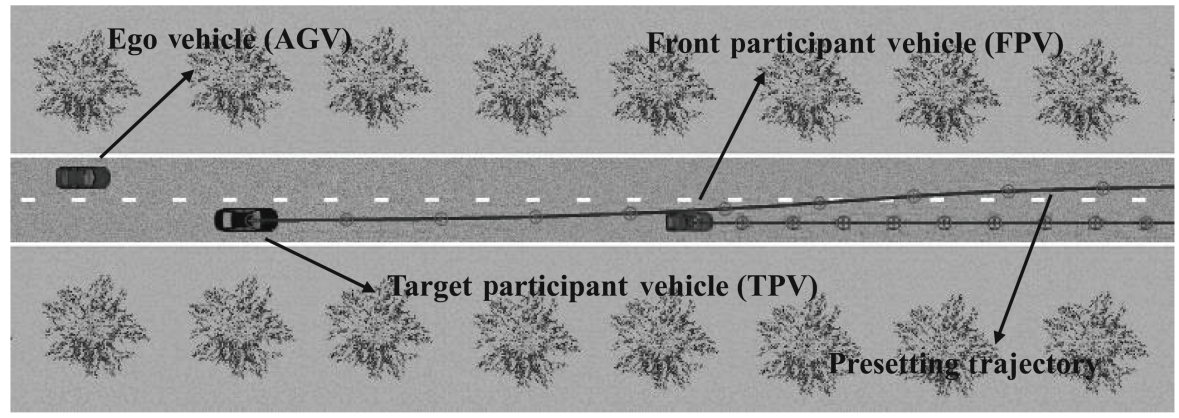

Figure 6. Scenario setup for accuracy comparison of prediction.

the prediction. In contrast, the predicted results of CVP and TKP seem to have no apparent consideration to avoid the collision. Moreover, we can find that the velocity profile predicted by IAP is most similar to the actual velocity profile at three time points.

(3) Quantitative Evaluation on Simulation: To compare the precision of three predictors within two different time spans (i.e., $[0,2] \mathrm{s}$ and $[0,3] \mathrm{s}$ ), the errors of the predicted position $\mathbb{P}_{\text {pre }}$ and the actual position $\mathbb{P}_{\text {act }}$ at three time points are calculated. The calculation equation is written as (17). $\tau$ is the time length of the time span (i.e., 2 and 3):

$$
\text { Error }_{t}=\sum_{k=0}^{\tau * 10}\left\|\mathbb{P}_{\text {pre }}^{t+k * 0.1}-\mathbb{P}_{a c t}^{t+k * 0.1}\right\|_{2}, t=1,2,3
$$

The mean and variance of errors are shown in Table 2. It can be seen that IAP has the least average mean of error among three predictors. The average prediction error of IAP within $[0,2] \mathrm{s}$ is $69.37 \%, 29.18 \%$ of that 


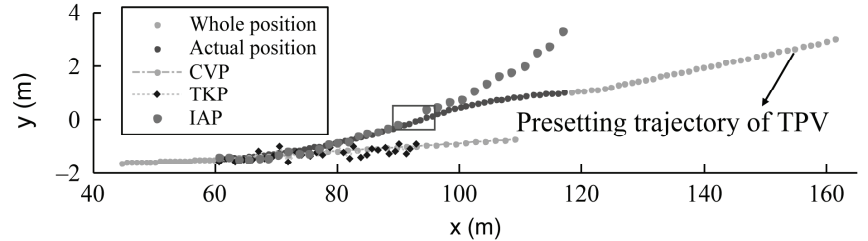

(a)

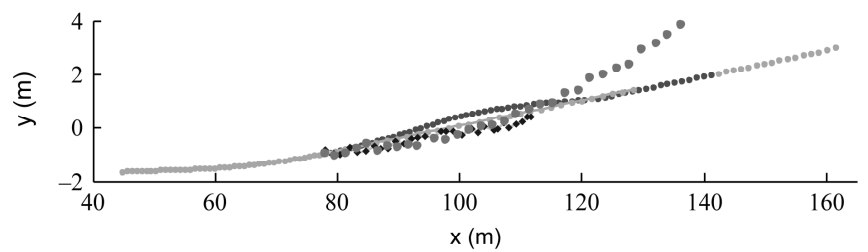

(b)

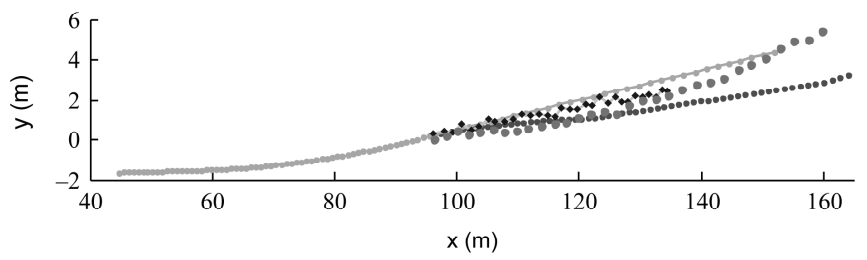

(c)

Figure 7. Comparison of trajectory prediction at three time points: (a) trajectory prediction at 1st second; (b) trajectory prediction at 2nd second; and (c) trajectory prediction at 3 rd second.

of CVP and TKP. The average prediction error of IAP within $[0,3] \mathrm{s}$ is $66.96 \%, 26.72 \%$ of that of CVP and TKP. All predictors perform the best at the beginning of the lane change. As time goes on, the errors grow larger. However, all errors of prediction within $[0,2] \mathrm{s}$ are less than those within $[0,3] \mathrm{s}$, except the prediction by IAP at the 1 st second.

\subsection{Performance of Predictive Motion Planning}

We choose two typical scenarios (i.e., crowded street and cut-in manoeuvre) to compare the performance of proposed motion planner IAPMP with previous ILTP, which is a non-predictive motion planner. The predicted time span of the IAPMP is $2 \mathrm{~s}$. The main performance metrics chosen are planned steering angle, planned velocity and the curvature of the executed trajectory. Note that the steering angle is denoted as negative when it is on the right-hand side from its centre and positive for the left-hand side.

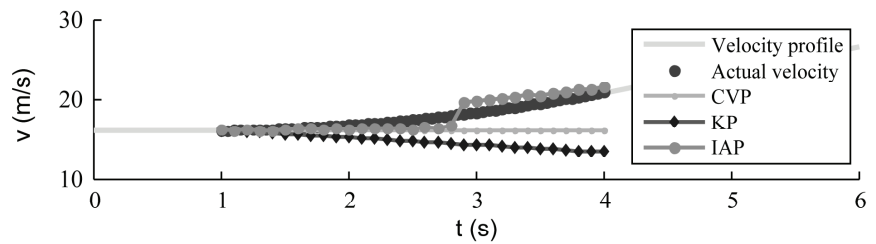

(a)

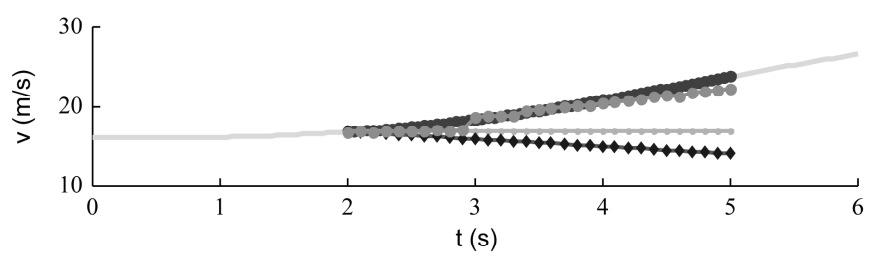

(b)

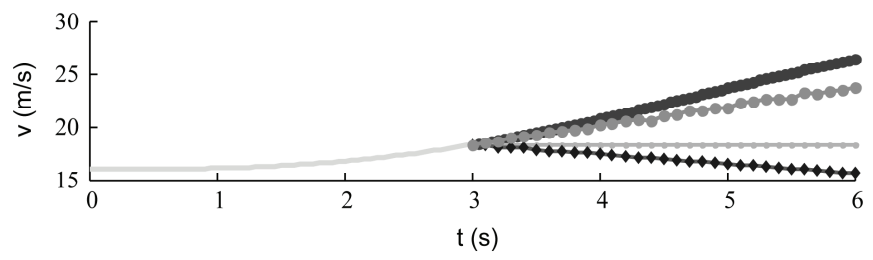

(c)

Figure 8. Comparison of velocity prediction at three time points: (a) velocity prediction at 1st second; (b) velocity prediction at $2 \mathrm{nd}$ second; and (c) velocity prediction at $3 \mathrm{rd}$ second.

The first scenario is where our UGV drives on a oneway, double-lane road with two preceding PVs of different velocities. PV\#1 has a higher velocity and overtakes PV\#2 during the simulation. The scenario and velocity profiles of two PVs are shown in Fig. 9(a)-(c).

The comparative experimental results between IAPMP and ILTP in a crowded street scenario are shown in the left column of Fig. 10. The planned trajectories, which are executed by the path follower, are shown in Fig. 10(a). For simplicity, the trajectories of PVs are not presented in the figure. Screenshots of driving in human view at specific time are shown in Fig. 10(b)-(g), which can be used to understand the situation intuitively. The trajectory planned by IAPMP is more human like than the one of ILTP, which changes lane to avoid PV\#1 in a short-sighted manner. The planned results of performance metrics are shown in Fig. 10(h)-(j). The planned steering angle of IAPMP is smoother than that of ILTP, and the curvature of the trajectory planned by IAPMP is also smoother. Meanwhile, IAPMP maintains a relatively less volatile planned velocity than that of ILTP.

Table 2

Accuracy of Trajectory Prediction

\begin{tabular}{|l|c|c|c|c|c|c|}
\hline \multirow{2}{*}{$\begin{array}{l}\text { Predicted } \\
\text { Time Point }\end{array}$} & \multicolumn{3}{|c|}{ Error $(\mathrm{m})$ in $[0,2]$} & \multicolumn{3}{c|}{ Error $(\mathrm{m})$ in $[0,3]$} \\
\cline { 2 - 7 } & CVP & TKP & IAP & CVP & TKP & IAP \\
\hline 1st second & $2.223 \pm 0.647$ & $6.171 \pm 3.404$ & $1.774 \pm 0.624$ & $3.284 \pm 1.763$ & $10.051 \pm 6.611$ & $1.579 \pm 0.695$ \\
\hline 2nd second & $3.001 \pm 1.257$ & $7.167 \pm 4.144$ & $1.914 \pm 0.216$ & $4.817 \pm 3.034$ & $11.896 \pm 8.046$ & $2.281 \pm 0.692$ \\
\hline 3rd second & $3.914 \pm 1.869$ & $8.388 \pm 4.924$ & $2.650 \pm 0.719$ & $6.321 \pm 4.041$ & $13.810 \pm 9.276$ & $3.610 \pm 1.614$ \\
\hline Average & $3.046 \pm 1.258$ & $7.242 \pm 4.157$ & $2.113 \pm 0.520$ & $4.807 \pm 2.946$ & $11.919 \pm 7.978$ & $2.490 \pm 1.000$ \\
\hline
\end{tabular}




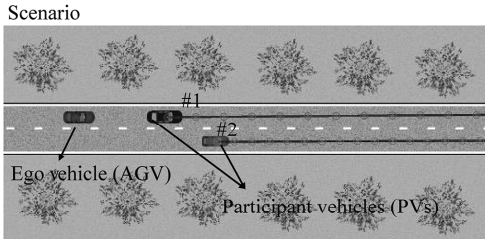

(a)

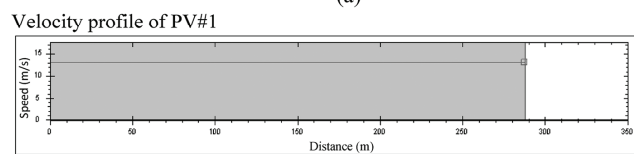

(b)

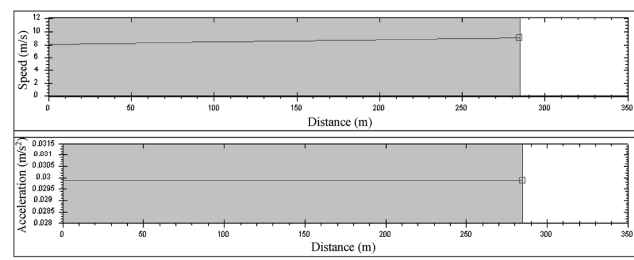

(c)
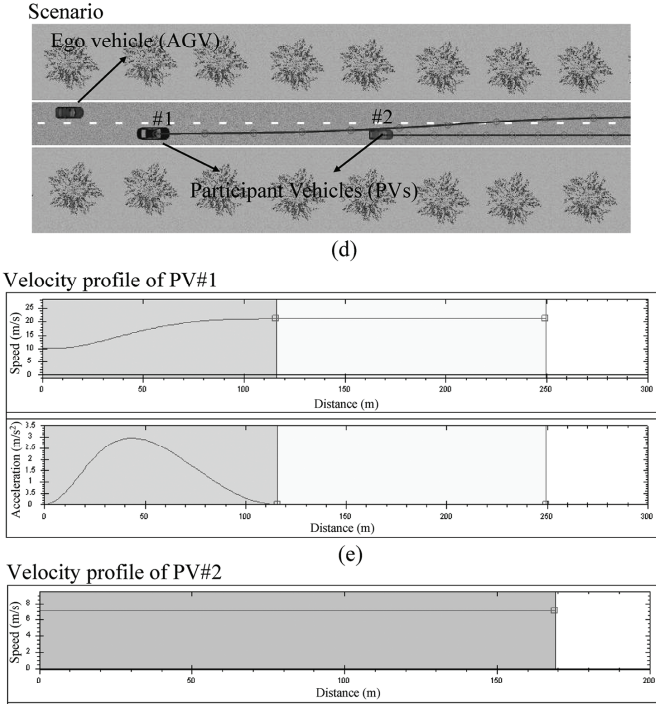

Figure 9. Left column: scenario 1 crowded street; right column: scenario 2 cut-in manoeuvre; (a) and (d) experimental scenario; (b), (c), (e) and (f) velocity profiles of PVs.

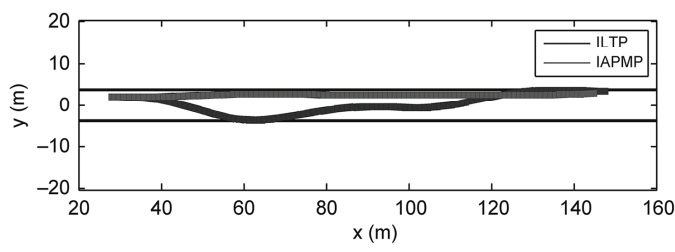

(a) Trajectory comparison

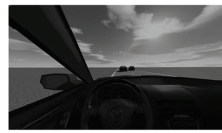

(b) IAPMP $1 \mathrm{~s}$

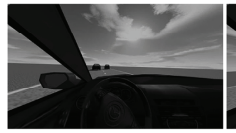

(e) ILT'P ls

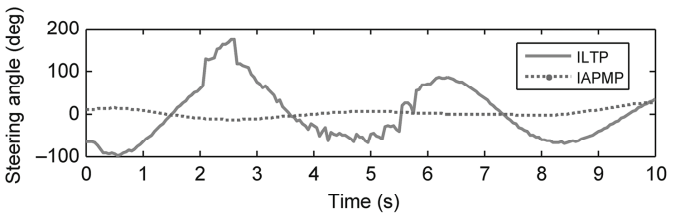

(h) Steering comparison

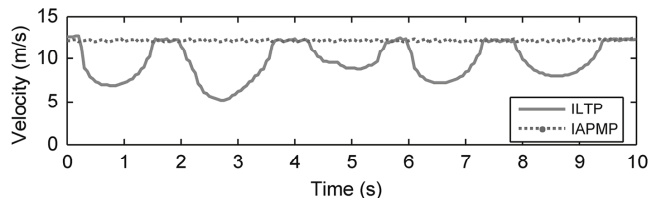

(i) Velocity comparison

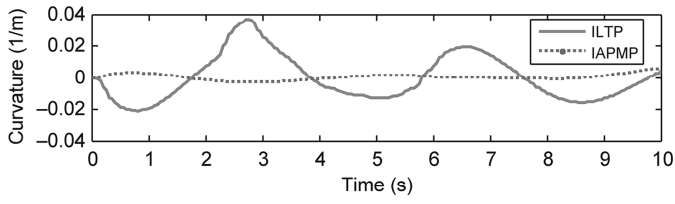

(j) Curvature comparison

(g) ILTP 6s

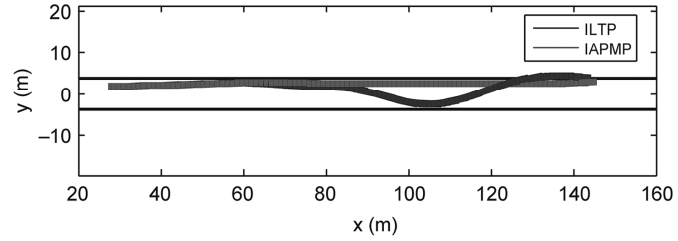

(k) Trajectory comparison
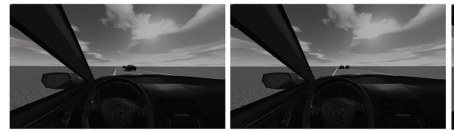

(m) IAPMP 5s

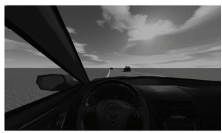

(1) IAPMP 3s
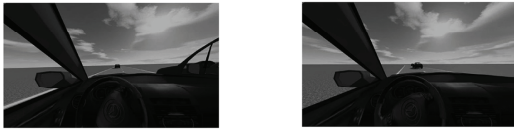

(o) ILTP 3s

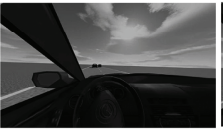

(p) ILTP 5s
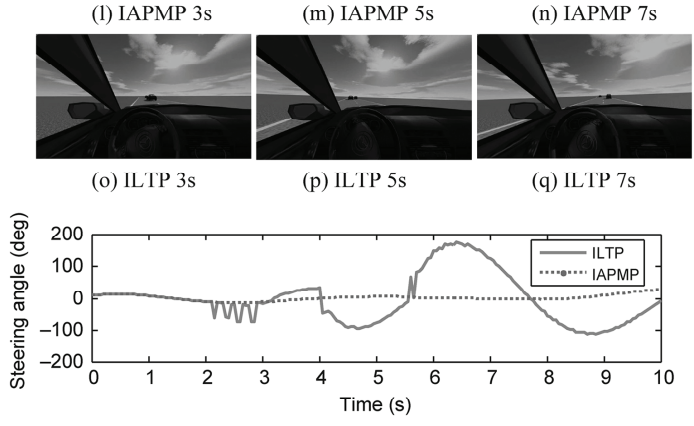

(r) Steering comparison

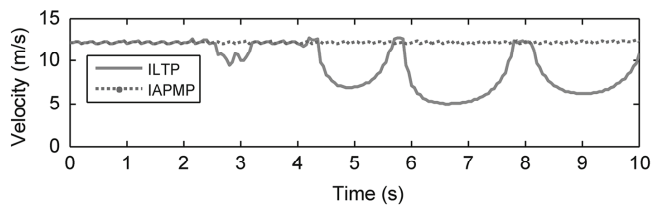

(s) Velocity comparison

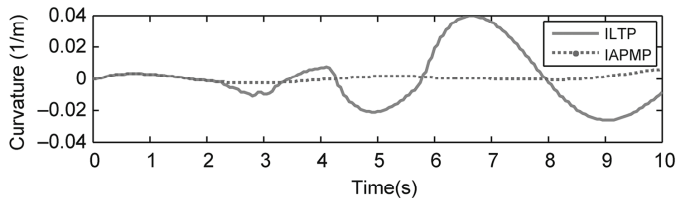

(t) Curvature comparison

Figure 10. Comparative results between IAPMP and ILTP. Left column: scenario 1 crowded street; Right column: scenario 2 cut-in manoeuvre; (a) and (k) graphs showing the trajectories of UGVs; (b)-(g) and (l)-(q) screenshots of driving in human view; $(\mathrm{h})-(\mathrm{j})$ and $(\mathrm{r})-(\mathrm{t})$ comparative results of performance metrics. 
The second scenario also occurs on a unidirectional double-lane road. PV\#1 overtakes PV\#2 and cuts into the lane occupied by the UGV. The scenario and velocity profiles of two PVs are shown in Fig. 9(d)-(f).

The results of two planners in a cut-in street scenario are shown in the right column of Fig. 10. The figures show that although the trajectory planned by ILTP is the same as IAPMP before $2 \mathrm{~s}$, the cut-in behaviour performed by PV\#1 will influence ILTP. As we can see in Fig. 10(r) and $(\mathrm{s})$, when the desired steering angle changes, the desired velocity also slows down correspondingly. In the cutin street scenario, the curvature of the trajectory planned by IAPMP is still smoother than that of ILTP.

As these two experiments demonstrate, when the UGV interacts with PVs in crowded and cut-in street scenarios, the smoothness of trajectories generated by IAPMP is improved compared to ILTP.

\section{Conclusion}

The presented motion planner IAPMP can interact with other moving PVs in some typical dynamic environments. The planned trajectories are collision-free, smooth and environmental adaptive. The performance of IAPMP in a dynamic environment has been greatly improved compared with the motion planner ILTP. The results also show that the proposed IAPMP is good at predicting the complex patterns in the long-term prediction.

A major challenge here is to meet the requirement of more complex manoeuvre predictions in real environment. This will be considered in future work. Besides, our motion planner will also be verified in an actual road test.

\section{Acknowledgement}

This research is supported by the National Key R\&D Program of China (Grant Number 2018YFB1305105) and the National Natural Science Foundation of China (Grant Numbers 61790565, U1564214 and 61751311).

\section{References}

[1] A. Geiger, P. Lenz, and R. Urtasun, Are we ready for autonomous driving? The Kitti vision benchmark suite, in 2012 IEEE Conf. on Computer Vision and Pattern Recognition (CVPR), IEEE, Washington, D.C, USA, 2012, 3354-3361.

[2] A. Broggi, M. Buzzoni, S. Debattisti, P. Grisleri, M.C. Laghi, P. Medici, and P. Versari, Extensive tests of autonomous driving technologies, IEEE Transactions on Intelligent Transportation Systems, 14(3), 2013, 1403-1415.

[3] F. Codevilla, M. Miiller, A. López, V. Koltun, and A. Dosovitskiy, End-to-end driving via conditional imitation learning, in 2018 IEEE Int. Conf. on Robotics and Automation (ICRA), IEEE, Brisbane, Queensland, Australia, 2018, 1-9.

[4] T.M. Howard, C.J. Green, A. Kelly, and D. Ferguson, State space sampling of feasible motions for high performance mobile robot navigation in complex environments, Journal of Field Robotics, 25(6-7), 2008, 325-345.

[5] R. Kala and K. Warwick, Motion planning of autonomous vehicles on a dual carriageway without speed lanes, Electronics, 4(1), 2015, 59-81.

[6] X. You, S. Liu, and C. Zhang, An improved ant colony system algorithm for robot path planning and performance analysis, International Journal of Robotics and Automation, 33(5), 2018.
[7] C. Lin, H. Wang, J. Yuan, and M. Fu, An online path planning method based on hybrid quantum ant colony optimization for AUV, International Journal of Robotics and Automation, 33(4), 2018.

[8] L. Yi, M. Cong, H. Dong, and D. Liu, Reinforcement learning and ega-based trajectory planning for dual robots, International Journal of Robotics and Automation, 33(4), 2018.

[9] S. Lefèvre, D. Vasquez, and C. Laugier, A survey on motion prediction and risk assessment for intelligent vehicles, ROBOMECH Journal, 1(1), 1, 2014.

[10] X. Li, Z. Sun, D. Cao, D. Liu, and H. He, Development of a new integrated local trajectory planning and tracking control framework for autonomous ground vehicles, Mechanical Systems and Signal Processing, 87(Part B), 2015, 118-137.

[11] D. Gonzalez, J. Perez, V. Milanes, and F. Nashashibi, A review of motion planning techniques for automated vehicles, IEEE Transactions on Intelligent Transportation Systems, 17(4), 2016, 1135-1145.

[12] M. Pivtoraiko, R.A. Knepper, and A. Kelly, Differentially constrained mobile robot motion planning in state lattices, Journal of Field Robotics, 26(3), 2009, 308-333.

[13] M. Likhachev and D. Ferguson, Planning long dynamically feasible maneuvers for autonomous vehicles, International Journal of Robotics Research, 28(8), 2009, 933-945.

[14] D. Fassbender, A. Mueller, and H.-J. Wuensche, Trajectory planning for car-like robots in unknown, unstructured environments, in 2014 IEEE/RSJ Int. Conf. on Intelligent Robots and Systems, IEEE, Chicago, Illinois, USA, 2014, 3630-3635.

[15] A.J. Rimmer and D. Cebon, Planning collision-free trajectories for reversing multiply-articulated vehicles, IEEE Transactions on Intelligent Transportation Systems, 17, 2016, 1998-2007.

[16] R. Cui, Y. Li, and W. Yan, Mutual information-based multiAUV path planning for scalar field sampling using multidimensional RRT, IEEE Transactions on Systems, Man, and Cybernetics: Systems, 46(7), 2016, 993-1004.

[17] M. Du, T. Mei, H. Liang, J. Chen, R. Huang, and P. Zhao, Drivers' visual behavior-guided RRT motion planner for autonomous on-road driving, Sensors (Switzerland), 16(1), 2016, $1-19$.

[18] B. Hao and Z. Yan, Recovery path planning for an agricultural mobile robot by Dubins-RRT* algorithm, International Journal of Robotics and Automation, 33(2), 2018.

[19] F. von Hundelshausen, M. Himmelsbach, F. Hecker, A. Mueller, and H.-J. Wuensche, Driving with tentacles: integral structures for sensing and motion, Journal of Field Robotics, 25(9), 2008, 640-673.

[20] S.M. Erlien, S. Fujita, and J.C. Gerdes, Shared steering control using safe envelopes for obstacle avoidance and vehicle stability, IEEE Transactions on Intelligent Transportation Systems, 17(2), 2016, 441-451.

[21] R. Zhao and D. Sidobre, On-line trajectory generation considering kinematic motion constraints for robot manipulators, International Journal of Robotics and Automation, 33(6), 2018.

[22] A. Kelly and B. Nagy, Reactive nonholonomic trajectory generation via parametric optimal control, The International Journal of Robotics Research, 22(7-8), 2003, 583-601.

[23] T.M. Howard and A. Kelly, Optimal rough terrain trajectory generation for wheeled mobile robots, The International Journal of Robotics Research, 26(2), 2007, 141-166.

[24] B. Nagy and A. Kelly, Trajectory generation for car-like robots using cubic curvature polynomials, 6th International Conference on Field and Service Robots, Helsinki, Finland, 11, 2001.

[25] M. Matthew, Parallel algorithms for real-time motion planning. Ph.D. Thesis, Carnegie Mellon University, Pittsburgh, PA, USA, 2011

[26] M. Werling, J. Ziegler, S. Kammel, and S. Thrun, Optimal trajectory generation for dynamic street scenarios in a Frenet frame, in 2010 IEEE Int. Conf. on Robotics and Automation (ICRA), IEEE, Anchorage, Alaska, 2010, 987-993.

[27] T. Gu and J.M. Dolan, On-road motion planning for autonomous vehicles, in Int. Conf. on Intelligent Robotics and Applications, Springer, 2012, 588-597.

[28] T. Gu, J.M. Dolan, and J. Lee, On-road trajectory planning for general autonomous driving with enhanced tunability, in Intelligent Autonomous Systems, 13, Springer, 2016, 247-261. 
[29] T. Gu, J.M. Dolan, and J. Lee, On-road trajectory planning for general autonomous driving with enhanced tunability, in Intelligent Autonomous Systems 13, Springer, 2014, 247-261.

[30] K. Kawabata, A trajectory generation method for mobile robot based on iterative extension-like process, Artificial Life and Robotics, 21(4), 2016, 500-509.

[31] L. Zhang, L. Sun, S. Zhang, and J. Liu, Trajectory planning for an indoor mobile robot using quintic Bézier curves, in 2015 IEEE Int. Conf. on Robotics and Biomimetics (ROBIO) IEEE, Zhuhai, China, 2015, 757-762.

[32] K.R. Simba, N. Uchiyama, and S. Sano, Real-time smooth trajectory generation for nonholonomic mobile robots using Bézier curves, Robotics and Computer-Integrated Manufacturing, 41, 2016, 31-42.

[33] X. Li, Z. Sun, D. Cao, Z. He, and Q. Zhu, Real-time trajectory planning for autonomous urban driving: framework, algorithms, and verifications, IEEE/ASME Transactions on Mechatronics, 21(2), 2016, 740-753.

[34] J. Li, B. Dai, X. Li, C. Li, and Y. Di, A real-time and predictive trajectory-generation motion planner for autonomous ground vehicles, in 2017 9th Int. Conf. onIntelligent Human-Machine Systems and Cybernetics (IHMSC), 2, IEEE, Hangzhou, China, 2017, 108-113.

[35] C. Fulgenzi, A. Spalanzani, and C. Laugier, Dynamic obstacle avoidance in uncertain environment combining PVOs and occupancy grid, in 2007 IEEE International Conference on Robotics and Automation, Roma, Italy, IEEE, 2007, 1610-1616.

[36] M. Brännström, E. Coelingh, and J. Sjöberg, Model-based threat assessment for avoiding arbitrary vehicle collisions, 11(3), 2010, 658-669.

[37] G. Xie, H. Gao, L. Qian, B. Huang, K. Li, and J. Wang, Vehicle trajectory prediction by integrating physics- and maneuverbased approaches using interactive multiple models, IEEE Transactions on Industrial Electronics, 65(7), (2018), 59996008.

[38] W. Xu, J. Pan, J. Wei, and J.M. Dolan, Motion planning under uncertainty for on-road autonomous driving, in 2014 IEEE Int. Conf. on Robotics \& Automation(ICRA), Hong Kong, China, 2014, 2507-2512.

[39] J. Schlechtriemen, A. Wedel, J. Hillenbrand, G. Breuel, and K.-d. Kuhnert, A lane change detection approach using feature ranking with maximized predictive power, in 2014 IEEE Intelligent Vehicles Symposium (IV), Ypsilanti, Michigan, USA, June 8-11, 2014, 108-114.

[40] H. Woo, Y. Ji, H. Kono, Y. Tamura, Y. Kuroda, T. Sugano, Y. Yamamoto, A. Yamashita, and H. Asama, Dynamic potential-model-based feature for lane change prediction, 2016 IEEE Int. Conf. on Systems, Man, and Cybernetics, SMC 2016, Budapest, Hungary, 2017, 838-843.

[41] D. Kasper, G. Weidl, T. Dang, G. Breuel, A. Tamke, A. Wedel, and W. Rosenstiel, Object-oriented Bayesian networks for detection of lane change maneuvers, Intelligent Transportation System Magazine, 4(1), 2012, 673-678.

[42] M. Liebner, M. Baumann, F. Klanner, and C. Stiller, Driver intent inference at urban intersections using the intelligent driver model, IEEE Intelligent Vehicles Symposium, Madrid, Spain, 2012, 1162-1167.

[43] S.B. Amsalu, A. Homaifar, A. Karimoddini, and A. Kurt, Driver intention estimation via discrete hidden Markov model, in 2017 IEEE Int. Conf. on Systems, Man, and Cybernetics (SMC), Banff, Canada, October 2017, 2712-2717.

[44] D.J. Phillips, T.A. Wheeler, and M.J. Kochenderfer, Generalizable intention prediction of human drivers at intersections, IEEE Intelligent Vehicles Symposium (IV), California, USA, 2017, 1665-1670.

[45] D. Lee, Y.P. Kwon, S. McMains, and J.K. Hedrick, Convolution neural network-based lane change intention prediction of surrounding vehicles for ACC, 2017 IEEE 20th International Conference on Intelligent Transportation Systems (ITSC), Yokohama, Japan, 2017, 1-6.

[46] T. Gindele, S. Brechtel, and R. Dillmann, A probabilistic model for estimating driver behaviors and vehicle trajectories in traffic environments, in IEEE Conf. on Intelligent Transportation Systems (ITSC), Madeira Island, Portugal, 2010, 1625-1631.
[47] M. Schreier, V. Willert, and J. Adamy, An integrated approach to maneuver-based trajectory prediction and criticality assessment in arbitrary road environments, IEEE Transactions on Intelligent Transportation Systems, 17(10), 2016, 2751-2766.

[48] M. Bahram, C. Hubmann, A. Lawitzky, M. Aeberhard, and D. Wollherr, A combined model- and learning-based framework for interaction-aware maneuver prediction, IEEE Transactions on Intelligent Transportation Systems, 17(6), 2016, 1538-1550.

[49] M. Bahram, A. Wolf, M. Aeberhard, and D. Wollherr, A prediction-based reactive driving strategy for highly automated driving function on freeways, in IEEE Intelligent Vehicles Symposium (IV), Michigan, USA, 2014, 400-406.

[50] S. Brechtel, T. Gindele, and R. Dillmann, Probabilistic decision-making under uncertainty for autonomous driving using continuous pomdps, in 17th Int. IEEE Conf. on Intelligent Transportation Systems (ITSC), Qingdao, China, 2014, 392-399.

[51] E. Galceran, A.G. Cunningham, R.M. Eustice, and E. Olson, Multipolicy decision-making for autonomous driving via changepoint-based behavior prediction: Theory and experiment, Autonomous Robots, 41(6), 2007, 1367-1382.

[52] A.G. Cunningham, E. Galceran, R.M. Eustice, and E. Olson, MPDM: Multipolicy decision-making in dynamic uncertain environments for autonomous driving, IEEE Conf. on Robotics and Automation (ICRA), Washington, USA, 2015 1670-1677.

[53] T. Lee and Y.J. Kim, Massively parallel motion planning algorithms under uncertainty using POMDP, International Journal of Robotics Research, 35(8), 2016, 928-942.

[54] M. Bahram, A. Lawitzky, J. Friedrichs, M. Aeberhard, and D. Wollherr, A game-theoretic approach to replanning-aware interactive scene prediction and planning, IEEE Transactions on Vehicular Technology, 65(6), 2016, 3981-3992.

[55] D. Lenz, T. Kessler, and A. Knoll, Tactical cooperative planning for autonomous vehicles using MCTS, IEEE Intelligent Vehicles Symposium (IV), Gothenburg, Sweden, 2016, 1-7.

[56] F. Damerow and J. Eggert, Risk-aversive behavior planning under multiple situations with uncertainty, 18th IEEE Conf. on Intelligent Transportation Systems, (ITSC), Canary Islands, Spain, September 2015, 656-663.

[57] J. Wei, J.M. Dolan, and B. Litkouhi, A prediction-and cost function-based algorithm for robust autonomous freeway driving, in 2010 IEEE Intelligent Vehicles Symposium (IV), IEEE, San Diego, CA, USA, 2010, 512-517.

[58] J. Wei, J.M. Dolan, and B. Litkouhi, Autonomous vehicle social behavior for highway entrance ramp management, IEEE Intelligent Vehicles Symposium (IV), Gold Coast, Australia, 201-207, 2013.

[59] J. Li, B. Dai, X. Li, X. Xu, and D. Liu, A dynamic Bayesian network for vehicle maneuver prediction in highway driving scenarios: Framework and verification, Electronics, 8(1), 2019, 40

[60] S. Sarkka, On unscented Kalman filtering for state estimation of continuous-time nonlinear systems, IEEE Transactions on Automatic Control,, 52(9), 2007, 1631-1641.

[61] T.M. Howard and A. Kelly, Optimal rough terrain trajectory generation for wheeled mobile robots, International Journal of Robotics Research, 26(2), 2007, 141-166.

[62] D. Ferguson, T.M. Howard, and M. Likhachev, Motion planning in urban environments, Journal of Field Robotics, 25(11-12), 2008, 939-960.

[63] R.A. Knepper and M.T. Mason, Real-time informed path sampling for motion planning search real-time informed path sampling for motion, International Journal of Robotics Research, 31(11), 2012, 1231-1250

[64] R.A. Knepper, S.S. Srinivasa, and M.T. Mason, Toward a deeper understanding of motion alternatives via an equivalence relation on local paths, International Journal of Robotics Research, 31(2), 2012, 167-186.

[65] J. Ziegler, P. Bender, T. Dang, and C. Stiller, Trajectory planning for Bertha-A local, continuous method, IEEE Intelligent Vehicles Symposium, Michigan, USA, Vol. 35, April, 2014, 450-457. 


\section{Biographies}

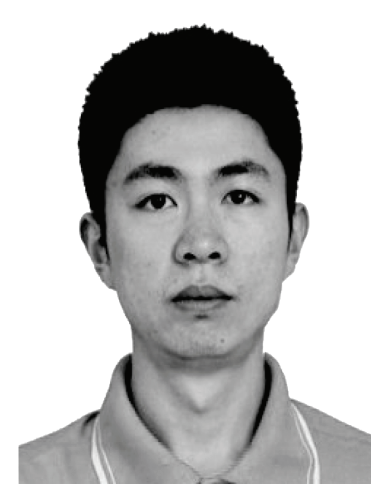

tic graph model.

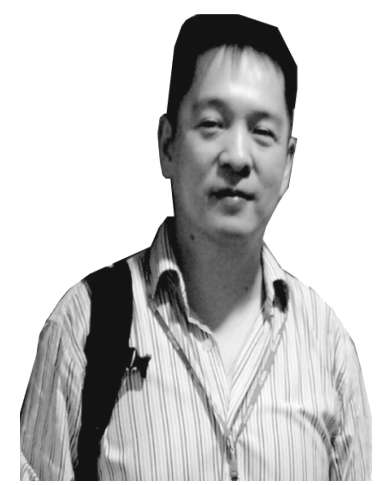

Bin Dai received Ph.D. degree in Control Science and Engineering from National University of Defense Technology, Changsha, P.R. China, in 1998. He was a visiting scholar with the Institute for Process Control and Robotics at Karlsruhe Institute of Technology in 2006. He is currently a professor with Unmanned Systems Research Center, National Innovation Institute of Defense Technology. His research interests include pattern recognition, computer vision and intelligent vehicles.

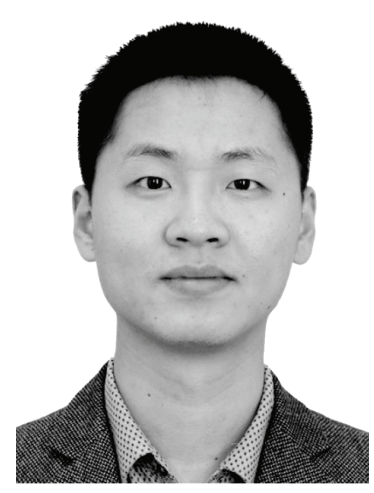

Xiaohui $L i$ is a lecturer in National University of Defense Technology (NUDT), P.R. China. He received the B.S. degree in electrical engineering from Harbin Institute of Technology, China, in 2009 and the Ph.D. degree in pattern recognition and intelligent system from NUDT in 2016. He was a visiting Ph.D. student at the Department of Electrical and Computer Engineering at the Ohio State University (OSU), USA, during 2013-2014. His research interests include autonomous driving, behavior reasoning, motion planning and motion control for intelligent systems.

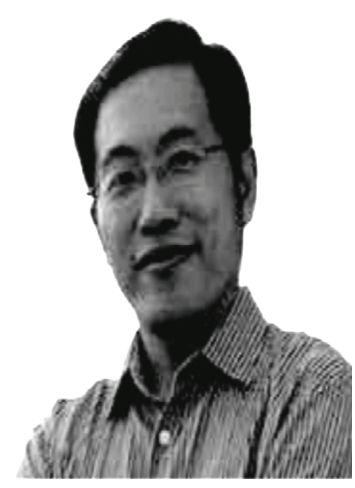

Ruili Wang received his B.Sc. degree from Huazhong University of Science and Technology, Wuhan, China, and M.Sc. degree from Northeastern University, Shenyang, China, and the Ph.D. degree from Dublin City University, Dublin, Ireland. Currently, he is the Professor of AI, Massey University, Auckland, New Zealand. His research interests include speech processing, language processing, image processing, intelligent systems and complex systems.

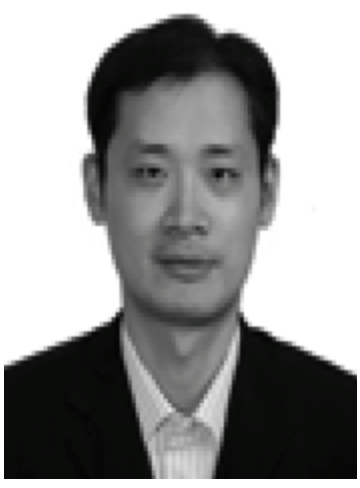

$X$ in $X u$ received the Ph.D. degree in control science and engineering from the College of Mechatronics and Automation, National University of Defense Technology (NUDT), in 2002. He has been a visiting professor in Hong Kong Polytechnic University, University of Alberta, University of Guelph, and the University of Strathclyde, UK. He is currently a full Professor with the Institute of Unmanned Systems, College of Intelligence Science and Technology, NUDT, China. He has co-authored more than 160 papers in international journals and conferences, and co-authored four books. His research interests include intelligent control, reinforcement learning, approximate dynamic programming, machine learning, robotics and autonomous vehicles. He received the National Science Fund for Outstanding Youth in China and the 2nd class National Natural Science Award of China. He has served as an Associate Editor or Guest Editor of Information Sciences, IEEE Transactions on Systems, Man and Cybernetics: Systems, Intelligent Automation and Soft Computing, International Journal of Adaptive Control and Signal Processing and Acta Automatica Sinica. $\mathrm{He}$ is a Member of the IEEE CIS Technical Committee on Approximate Dynamic Programming and Reinforcement Learning (ADPRL) and the IEEE RAS Technical Committee on Robot Learning.

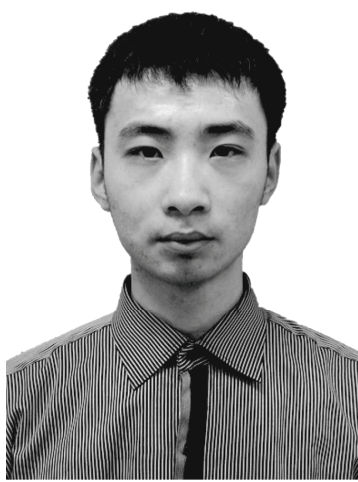

Bohan Jiang is currently pursuing his Ph.D. degree with the College of Intelligence Science and Technology at National University of Defense Technology, Changsha, China. He obtained his M.Sc. degree from Harbin Engineering University in 2014. His research interests include motion planning and semi-autonomous driving technology.

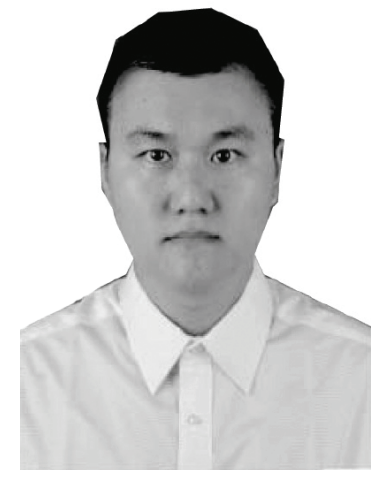

$Y i D i$ is currently a lecturer in Information Engineering School, Wuchang University of Technology. He obtained his Ph.D. degree from Nanjing University of Science and Technology in 2017, and the M. Sc. degree in signal processing from Guizhou University in 2013. His research interests include intelligent signal processing, target detection, recognition and tracking technology. 